Chapman University

Chapman University Digital Commons

Pharmacy Faculty Articles and Research

School of Pharmacy

8-1-2014

\title{
UHPLC-MS/MS Analysis of Arachidonic Acid and 10 of Its Major Cytochrome P450 Metabolites as Free Acids in Rat Livers: Effects of Hepatic Ischemia
}

Vindhya Edpuganti

Texas Tech University Health Sciences Center

Reza Mehvar

Chapman University, mehvar@chapman.edu

Follow this and additional works at: http://digitalcommons.chapman.edu/pharmacy_articles

Part of the Animals Commons, Animal Structures Commons, Digestive System Commons, and the Organic Chemicals Commons

\section{Recommended Citation}

Edpuganti, V and Mehvar R, UHPLC-MS/MS Analysis of Arachidonic Acid and 10 of Its Major Cytochrome P450 Metabolites as Free Acids in Rat Livers: Effects of Hepatic Ischemia. J. Chromatogr. B Analyt. Technol. Biomed. Life Sci. 964: 153-163 (2014). doi: 10.1016/j.jchromb.2013.08.008

This Article is brought to you for free and open access by the School of Pharmacy at Chapman University Digital Commons. It has been accepted for inclusion in Pharmacy Faculty Articles and Research by an authorized administrator of Chapman University Digital Commons. For more information, please contact laughtin@chapman.edu. 


\section{UHPLC-MS/MS Analysis of Arachidonic Acid and 10 of Its Major Cytochrome P450 Metabolites as Free Acids in Rat Livers: Effects of Hepatic Ischemia}

\section{Comments}

NOTICE: this is the author's version of a work that was accepted for publication in Journal of Chromatography $B$. Changes resulting from the publishing process, such as peer review, editing, corrections, structural formatting, and other quality control mechanisms may not be reflected in this document. Changes may have been made to this work since it was submitted for publication. A definitive version was subsequently published in Journal of Chromatography B, volume 964, in 2014. DOI: 10.1016/j.jchromb.2013.08.008

The Creative Commons license below applies only to this version of the article.

\section{Creative Commons License}

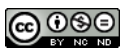

This work is licensed under a Creative Commons Attribution-Noncommercial-No Derivative Works 4.0 License.

\section{Copyright}

Elsevier 


\title{
UHPLC-MS/MS analysis of arachidonic acid and 10 of its major cytochrome P450 metabolites as free acids in rat livers: Effects of hepatic ischemia
}

\author{
Vindhya Edpuganti ${ }^{\mathrm{a}}$, Reza Mehvar ${ }^{\mathrm{a},{ }^{*}}$ \\ ${ }^{a}$ Department of Pharmaceutical Sciences, School of Pharmacy, Texas Tech University Health \\ Sciences Center, Amarillo, Texas, USA
}

* Corresponding author at: Department of Pharmaceutical Sciences, School of Pharmacy, Texas Tech University Health Sciences Center, 1300 Coulter, Amarillo, TX 79106, USA; Phone: (806) 356-4015 Ext. 337; FAX: (806) 356-4034; E-mail: reza.mehvar@ttuhsc.edu. 


\section{ABSTRACT}

The cytochrome P450 metabolites of arachidonic acid (AA) are mostly present in tissues, such as the liver, as bound to phospholipids, with only a small fraction available as free acids. The purpose of this study was to develop and validate a UHPLC-MS/MS method for quantitation of free liver concentrations of AA and four epoxygenated (5,6-, 8,9-, 11,12-, and 14,15-EET), four dihydroxylated (5,6-, 8,9-, 11,12-, and 14,15-DHET), and two $\omega /(\omega-1)$ hydroxylated (19- and 20HETEs) metabolites of AA in rat livers using deuterated internal standards. The analytes were rapidly and efficiently (79-92\%) recovered from $100 \mathrm{mg}$ of fresh liver into methanol. After evaporation, the reconstituted samples were injected either undiluted (for the simultaneous analysis of the metabolites) into a gradient or diluted (for AA analysis) into an isocratic UHPLC system with run times of 5 and 2 min, respectively. Mass spectrometry was conducted using multiple reaction monitoring in negative mode. The method was linear $\left(r^{2} \geq 0.98\right)$ in the concentration ranges tested for metabolites (0.19-120 ng/g liver) and AA (7.8-500 $\mu \mathrm{g} / \mathrm{g}$ liver). The lower limit of quantitation of the assay was between 0.57 to $5.6 \mathrm{pg}$ injected on column for different AA metabolites. The assay was validated $(n=5)$ based on acceptable intra- and interrun accuracy and precision values. Additionally, matrix effect was minimal for most analytes. Freeze-thaw of samples drastically increased the free liver concentrations of analytes, presumably due to their release from the membrane storage sites. Therefore, fresh liver samples should be used for analysis. However, the methanolic extracts may be stored at $-80^{\circ} \mathrm{C}$ for at least two weeks without any compromise. The method was successfully used in the measurement of all the analytes in the rats subjected to $60 \mathrm{~min}$ of hepatic ischemia $(n=6)$ or sham operation $(n=$ 6). Ischemia resulted in significantly higher free concentrations of AA and most of its studied metabolites. The method is precise, accurate, and sensitive for measurement of free liver concentrations of AA and its P450 metabolites in the rat liver. 
Keywords: Arachidonic acid; Cytochrome P450 metabolites; UHPLC-MS/MS; Liver; Ischemia 


\section{Introduction}

Arachidonic acid (AA), or 5,8,11,14-eicosatetraenoic acid, is a polyunsaturated omega-6 fatty acid that is abundantly present in the phospholipids of cellular membranes. AA is released from membranes upon hydrolysis of the $s n-2$ fatty acyl bond of phospholipids by activation of calcium dependent type IV phospholipase $A_{2}$ in response to stimulus $[1,2]$. The released AA can be subsequently metabolized by three different groups of enzymes, namely cyclooxygenases, lipoxygenases, and cytochromes P450 (P450) [2-5]. Whereas the cyclooxygenase enzyme converts AA to prostaglandins and thromboxane $\mathrm{A}_{2}$, the products of $\mathrm{AA}$ metabolism by lipoxygenase are 5-, 8-, 12-, and 15-hydroxyeicosatetraenoic acids (HETEs) and leukotrienes [5]. The more recently-discovered metabolic pathway catalyzed by P450 enzymes [6-8] includes epoxygenation of AA to four regioselective epoxyeicosatrienoic acids (EETs) and $\omega /(\omega-1)$ hydroxylation to two major hydroxylated metabolites 19- and 20-HETE (Fig. 1) in addition to other regioselective hydroxylated metabolites. Similar to AA, EETs are also extensively incorporated into the $s n-2$ position of phospholipids, and only a small fraction of EETs $(<1 \%)$ are in the form of free acids in the tissues $[9,10]$. Additionally, EETs are further metabolized by soluble epoxide hydrolase (sEH) to dihydroxyeicosatrienoic acids (DHETs) (Fig. 1) [10]. Therefore, the concentrations of free EETs in tissues are very low [9].

Both hydroxylated and epoxygenated P450 metabolites of AA are known to be potent regulators of vascular tone. For example, in coronary circulation, it is believed that HETEs (mainly 20-HETE) act as vasoconstrictors [11], whereas EETs act as vasodilators [12]. However, these effects may be tissue dependent because 20-HETE induces medullary vasodilation in the kidneys [13]. Because of their effects on the vascular tone, and possibly other cellular events, EETs and HETEs play a significant role in the pathophysiology of different diseases, such as 
ischemia-reperfusion (IR) injury [14] and portal hypertension [15]. Therefore, it is necessary to study the disposition and effects of P450-mediated metabolites of AA in different diseases.

The study of the disposition of the P450-mediated metabolites of AA requires sophisticated analytical methods capable of simultaneous quantitation of regioselective isomers of epoxygenated (EETs), hydroxylated (HETEs), and dihydroxylated (DHETs) metabolites with very low free concentrations in the plasma and tissues. In the past, techniques like gas chromatography-mass spectrometry (GC-MS) [16, 17], liquid chromatography (LC) with fluorescence detection [18, 19], LC-mass spectrometry (LC-MS) [20, 21], and LC-tandem mass spectrometry (LC-MS/MS) [22-24] were used to measure the free concentrations of some EETs, HETEs, or DHETs in plasma or tissue samples. Among these methods, LC-MS/MS has the highest potential in this area due to its high specificity and sensitivity for simultaneous quantitation of a wide range of structurally similar analytes, such as structural isomers of eicosanoids, within a single run [25]. However, to the best of our knowledge, a validated LCMS/MS method for quantitation of free concentrations of EETs, HETEs, and DHETs in the liver tissue is not yet available. Such a method is especially important because despite the fact that the liver is the major site of P450 enzymes in the body, the disposition and effects of P450-mediated AA metabolites in the liver are largely unknown. Therefore, the objective of the current study was to develop and validate an accurate, sensitive, and reproducible method to simultaneously measure the free concentrations of AA and its major P450 metabolites in the liver matrix. Additionally, the method was successfully applied to evaluation of the effects of hepatic ischemia on the free liver concentrations of AA and its P450 metabolites in rats. 


\section{Materials and methods}

\subsection{Chemicals}

The following standards were purchased from Cayman Chemical Company (Ann Arbor, MI): 5Z,8Z,11Z,14Z-eicosatetraenoic acid or AA, 19-(R)-hydroxyeicosatetraenoic acid (19HETE), 20-hydroxyeicosatetraenoic acid (20-HETE), ( \pm )-5,6-epoxyeicosatrienoic acid (5,6EET), $( \pm)$-8,9-epoxyeicosatrienoic acid (8,9-EET), $( \pm)$-11,12-epoxyeicosatrienoic acid $(11,12-$ EET), ( $( \pm)$-14,15-epoxyeicosatrienoic acid (14,15-EET), ( \pm )-5,6-dihydroxyeicosatrienoic acid (5,6-DHET), ( $( \pm)-8,9$-dihydroxyeicosatrienoic acid (8,9-DHET), $( \pm)-11,12$ dihydroxyeicosatrienoic acid (11,12-DHET), and ( \pm )-14,15-dihydroxyeicosatrienoic acid (14,15DHET). Additionally, the following deuterated internal standards (IS) were also obtained from the same company: 20-HETE- $\mathrm{d}_{6}$ (deuterium atoms at the 16, 16', 17, 17', 18, and 18' positions; isotopic purity of $\geq 99 \%$ ), 8,9-EET-d d $_{11}$ (deuterium atoms at the 16,16',17, 17', 18, 18', 19, 19', 20, 20, and 20 positions; isotopic purity of $\geq 99 \%$ ), 14,15-EET- $\mathrm{d}_{11}$ (deuterium atoms at the 16 , $16 ', 17,17^{\prime}, 18,18^{\prime}, 19,19^{\prime}, 20,20$, and 20 positions; isotopic purity of $\left.\geq 99 \%\right), 14,15-$ DHET-d $\mathrm{d}_{11}$

(deuterium atoms at the 16, 16', 17, 17', 18, 18', 19, 19', 20, 20, and 20 positions; isotopic purity of $\geq 99 \%$ ), and $A A-d_{8}$ (deuterium atoms at the $5,6,8,9,11,12,14$, and 15 positions; isotopic purity of $\geq 99 \%$ ). LC-MS grade water was purchased under the brand name J.T. Baker from Avantor Performance Materials, Inc. (Center Valley, PA). HPLC grade glacial acetic acid and LCMS grade acetonitrile were purchased from Fisher Scientific (Fair Lawn, NJ). All other reagents used were also of HPLC grade and purchased through commercial sources.

\subsection{UHPLC-MS/MS instrumentation}

The UHPLC-MS/MS instrument consisted of an AB SCIEX QTRAP® 5500 mass spectrometer (Foster City, CA, USA) attached to a Nexera UHPLC system from Shimadzu 
Corporation (Columbia, MD). The UHPLC system consisted of a Sil-30AC autosampler, LC30AD pumps, a CBM-20A controller, a DGA-20 $\mathrm{A}_{5}$ degasser, and a CTO-30A column oven. Analyst and MultiQuant software were used for data acquisition and quantitation, respectively.

\subsection{Chromatographic conditions}

The chromatographic separation of analytes was performed at $40^{\circ} \mathrm{C}$ on a Kinetex $\mathrm{C} 18$ column ( $5 \mathrm{~cm}$ x $2.10 \mathrm{~mm}$ I.D., $1.7 \mu \mathrm{m}$ particle size, $100 \AA$ pore size $)$, preceded by a SecurityGurad ULTRA guard column (Phenomenex; Torrance, CA, USA). The elution of all the analytes, except for AA and AA- $\mathrm{d}_{8}$, was based on a gradient method using mobile phases of water: acetonitrile: acetic acid (95:5:0.005, v/v/v) (A) and acetonitrile: acetic acid (100:0.005, $\mathrm{v} / \mathrm{v}$ ) (B), which was run at a flow rate of $0.5 \mathrm{~mL} / \mathrm{min}$. The elution started with $50 \% \mathrm{~B}$, which was increased to $60 \%$ B over 2 min and kept constant until $3.2 \mathrm{~min}$. At $3.2 \mathrm{~min}$, the mobile phase was switched to $100 \%$ B and maintained until 4 min, which was followed by return to the initial condition. The total run time was $5 \mathrm{~min}$, but the mass spectrometer data was collected only from 0.4 to $3.1 \mathrm{~min}$. For AA and AA- $d_{8}$, an isocratic mobile phase of A:B $(20: 80, v / v)$ was used at a flow rate of $0.5 \mathrm{~mL} / \mathrm{min}$ for $2 \mathrm{~min}$. The mass spectrometer data here was collected from 0.3 to $1.5 \mathrm{~min}$. The injection volumes used were $3 \mu \mathrm{L}$ for all the samples because larger injection volumes caused band broadening.

\subsection{Mass spectrometric conditions}

A triple quadrupole mass spectrometer was used to analyze the analytes by monitoring their $m / z$ transitions with the help of Analyst software. The ionization source was through electrospray ionization (TurbolonSpray), and the analytes were detected using multiple reaction monitoring (MRM) operated in the negative mode. The source/gas and compound parameters 
were optimized to obtain the highest $[\mathrm{M}-\mathrm{H}]^{-}$ion abundance by infusing the standard solutions of the analyte of interest via a syringe pump into the mass spectrometer. The optimized source/gas parameters were as follows: curtain gas, 35 psi; collision gas, high; ion spray voltage, -4500 V, temperature, $650^{\circ} \mathrm{C}$, ion source gas 1 (nebulizer gas), $60 \mathrm{psi}$; and ion source gas 2 (turbo gas), 60 psi. The compound parameters were optimized for each of the analytes and are represented in Table 1. For most of the analytes, the product ions selected in our study (Table 1) are similar to those reported previously $[25,26]$.

\subsection{Preparation of stock solutions, calibration standards, and quality control samples}

All the stock solutions were prepared in ethanol and stored at $-80^{\circ} \mathrm{C}$. The stock solution of metabolites consisted of $60 \mathrm{ng} / \mathrm{mL}$ of 19-HETE, 20-HETE, and 11,12-DHET; $48 \mathrm{ng} / \mathrm{mL}$ of 8,9-EET and 8,9-DHET; $24 \mathrm{ng} / \mathrm{mL}$ of 5,6-EET, 11,12-EET, 14,15-EET, and 5,6-DHET; and 240 $\mathrm{ng} / \mathrm{mL}$ of 14,15-DHET. The stock solution of IS for the metabolites contained $60 \mathrm{ng} / \mathrm{mL}$ of 20 HETE-d $_{6}$ (for 19-HETE and 20-HETE), $50 \mathrm{ng} / \mathrm{mL}$ of 8,9-EET-d ${ }_{11}$ (for 8,9-EET and 11,12-EET), $50 \mathrm{ng} / \mathrm{mL}$ of 14,15-EET-d ${ }_{11}$ (for 5,6-EET and 14,15-EET), and $120 \mathrm{ng} / \mathrm{mL}$ of 14,15-DHET-d 11 (for 5,6-DHET, 8,9-DHET, 11,12-DHET, and 14,15-DHET). Calibration standards were then prepared by diluting the metabolite stock solution in ethanol before the addition of an equal volume of the IS solution. The resulting calibration standards ranged from $0.94-30 \mathrm{ng} / \mathrm{mL}$ for 19 HETE and 20-HETE; $0.48-30 \mathrm{ng} / \mathrm{mL}$ for 11,12-DHET; $0.75-24 \mathrm{ng} / \mathrm{mL}$ for 8,9-EET; 0.38-24 $\mathrm{ng} / \mathrm{mL}$ for 8,9-DHET; 0.38-12 ng/mL for 5,6-EET, 11,12-EET, and 14,15-EET; 0.19-12 ng/mL for 5,6-DHET; and 1.88-120 ng/mL for 14,15-DHET. A separate stock solution of AA consisting of $1000 \mu \mathrm{g} / \mathrm{mL}$ was prepared in ethanol. Calibration standards for AA were then prepared by diluting the stock solution with ethanol before mixing each standard with an equal volume of the IS solution $\left(140 \mu \mathrm{g} / \mathrm{mL}\right.$ of AA- $\left.\mathrm{d}_{8}\right)$. The resulting calibration standards for AA ranged from 7.81 
to $500 \mu \mathrm{g} / \mathrm{mL}$. Calibration curves were constructed by plotting the analyte: IS peak area ratios (y) against the concentration of each analyte (x) using a weight of 1/concentration. The unknown concentrations of analytes in the reconstituted final solution $(\mathrm{ng} / \mathrm{mL})$ injected into the instrument were then estimated from the calibration curve. Because in our studies we used $100 \mathrm{mg}$ of liver and reconstituted the final residue in $100 \mu \mathrm{L}$ of ethanol before injection (section 2.6), the estimated concentration in the reconstituted solution $(\mathrm{ng} / \mathrm{mL})$ was assumed to be the concentration in the liver tissue $(\mathrm{ng} / \mathrm{g})$.

Quality control (QC) samples were prepared in ethanol using the highest and lowest concentrations used in the calibration curves plus a third sample in the middle of the calibration curves (eight fold lower than the highest standard). These concentrations were 30, 3.75, and 0.94 $\mathrm{ng} / \mathrm{mL}$ for 19-HETE and 20-HETE; 12, 1.5, and $0.38 \mathrm{ng} / \mathrm{mL}$ for 5,6-EET, 11,12-EET, and 14,15EET; 24,3 , and $0.75 \mathrm{ng} / \mathrm{mL}$ for 8,9-EET; $12,1.5$, and $0.19 \mathrm{ng} / \mathrm{mL}$ for 5,6-DHET; $24,3,0.38$ $\mathrm{ng} / \mathrm{mL}$ for 8,9 -DHET; $30,3.75$, and $0.48 \mathrm{ng} / \mathrm{mL}$ for 11,12-DHET; 120,15 , and $1.88 \mathrm{ng} / \mathrm{mL}$ for 14,15-DHET; and 500, 62.5, and $7.81 \mu \mathrm{g} / \mathrm{mL}$ for AA.

\subsection{Sample preparation}

After addition of $950 \mu \mathrm{L}$ of ice-cold 0.01 M BHT in methanol, fresh liver samples (100 $\mathrm{mg}$ ) were homogenized using a glass/PTFE Potter Elvehjem tissue grinder for $15 \mathrm{~s}$. Subsequently, $50 \mu \mathrm{L}$ of the IS solution was added to the homogenate, and the samples were vortex-mixed for $5 \mathrm{~s}$ and centrifuged at $19,500 \mathrm{x}$ for $15 \mathrm{~min}$ at $0^{\circ} \mathrm{C}$. The resultant supernatant was transferred to a silanized glass tube and dried under nitrogen gas. The dried residue was reconstituted in $100 \mu \mathrm{L}$ of ethanol, vortex-mixed for $15 \mathrm{~s}$, and subjected to a final centrifugation at $19,500 \mathrm{x} g$ for $15 \mathrm{~min}$ at $0^{\circ} \mathrm{C}$. The resultant supernatant was divided into two parts for separate analysis of AA and the rest of the analytes. For the AA analysis, $10 \mu \mathrm{L}$ of the supernatant was 
diluted 4000 fold before injection into the UHPLC-MS/MS. The rest of the supernatant was used directly without dilution for the analysis of the other analytes. The injection volume was 3 $\mu \mathrm{L}$ in both cases.

\subsection{Method validation}

Intra- and inter-run accuracy and precision of the method were evaluated based on the analysis of QC samples, along with calibration standards, at low, middle, and high analyte concentrations $(n=5)$. Accuracy was calculated using the following equation:

$$
\text { Accuracy }(\%)=\frac{\text { Measured Concentration }}{\text { Nominal Concentration }} \times 100
$$

Precision was estimated as percentage relative standard deviation (R.S.D.). The acceptable limits for accuracy were $85 \%-115 \%$ for the middle and high concentrations and $80 \%-120 \%$ for the low concentration. The acceptable precision was $\leq 15 \%$ for the middle and high concentrations and $\leq$ $20 \%$ for the lower limit of quantitation (LLOQ).

The linearity of the method was evaluated by linear regression analysis of the calibration curves in the ranges stated in section 2.5.

\subsection{Recovery}

The recoveries of AA, metabolites, and internal standards from the liver homogenates were estimated by comparison of detector response to the processed liver homogenates (Baseline, $n=6$ ), homogenates spiked with known quantities of the analytes before processing (Test, $n=6$ ), and processed homogenates that were spiked with equivalent quantities of the analytes during the final reconstitution of the extracts (Control, $n=6)$. The recovery experiments were conducted at equivalent liver concentrations (spiked) of $15 \mathrm{ng} / \mathrm{g}$ of 19-HETE and 20- 
HETE; 12 ng/g of 5,6-EET; 6 ng/g of 8,9-EET, 11,12-EET, 14,15-EET, 11,12-DHET, and 14,15-DHET; $24 \mathrm{ng} / \mathrm{g}$ of 5,6-DHET and 8,9-DHET; and $500 \mu \mathrm{g} / \mathrm{g}$ of AA. The liver samples were spiked with the analytes after homogenization in methanol. The recoveries of internal standards were estimated at the concentrations that they were used in the assay. The following equation was used for the estimation of recoveries:

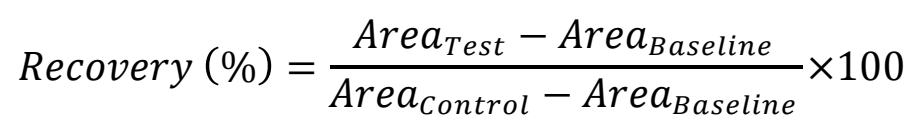

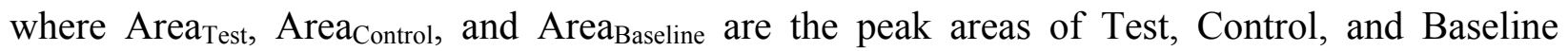

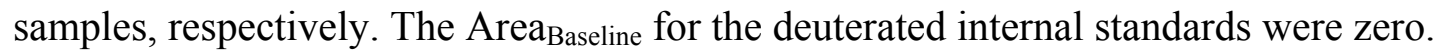

\subsection{Matrix effect}

Duplicate liver samples from six different rats were extracted into methanol, and the supernatants were evaporated. One set of samples was processed normally by dissolving the residues in $100 \mu \mathrm{L}$ of ethanol without analyte spiking (Baseline), whereas the other set was reconstituted in ethanol spiked with known quantities of the analytes (Matrix). Additionally, equivalent concentrations of analytes in ethanol (Standard) were directly injected into the UHPLC system without addition to any sample residue. The matrix factor (MF) was then calculated according to the following equation:

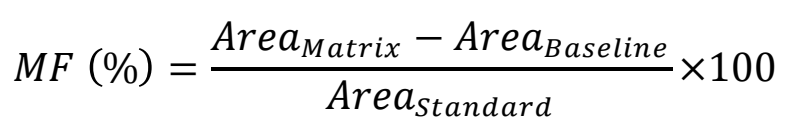

where Area $_{\text {Matrix, }}$ Area Baseline, and Area $_{\text {Standard }}$ are the peak areas of Matrix, Baseline (endogenous), and Standard samples, respectively. The Area Baseline for the deuterated internal standards was zero. Using the above equation, MF values less than $100 \%$ indicate ion 
suppression, values greater than $100 \%$ indicate ion enhancement, and values equal to $100 \%$ indicate no matrix effect.

\subsection{Effects of sample handling on the liver concentrations of AA and its metabolites}

Rat liver samples were subjected to the Sample preparation procedure described in section 2.6 immediately after collection (Fresh-0), after 20 (Fresh-20), 40 (Fresh-40), or 60 (Fresh-60) min of storage on ice, or after snap-freezing in nitrogen and thawing (20 min). The effects of sample handling method on the concentrations of AA and its metabolites were then determined $(n=3-4)$.

\subsection{Stability of the methanolic extracts of the processed fresh liver samples at $-80^{\circ} \mathrm{C}$}

The stability of the methanolic supernatants, which were obtained after homogenization of the fresh liver samples, was studied at $-80^{\circ} \mathrm{C}$ over a period of four weeks. Fresh liver samples $(n=6)$ from Sham rats (section 2.13) were homogenized in methanol as described in section 2.6, and the supernatants were stored at $-80^{\circ} \mathrm{C}$ without further processing. At baseline and after 1,2 , and 4 weeks of storage, the methanolic supernatants were further processed as described in section 2.6 and injected into the UHPLC-MS/MS instrument.

\subsection{Sample stability in the Autosampler}

The autosampler stability $\left(4^{\circ} \mathrm{C}\right)$ of AA, its metabolites, and internal standards in the processed liver samples $(n=4)$ was tested by injecting the samples into the UHPLC-MS/MS immediately (time zero) or after 1,3 , or $5 \mathrm{~h}$ of storage in the autosampler. The stability was evaluated by examining the absolute peak areas of the analytes and internal standards in addition to the analyte: IS peak area ratios after different autosampler storage times. 


\subsection{Application of the method}

The method was used to study the effects of injury from partial (70\%) hepatic ischemia (Ischemia) on the concentrations of AA and its metabolites in the rat livers. Adult male SpragueDawley rats (body weights ranging from 250-300 g) were purchased from Charles River Laboratories, Inc. (Wilmington, MA, USA) and maintained on a $12 \mathrm{~h}$ light/dark cycle in our institutional animal facility with free access to food and water before the experiments. All the procedures were in compliance with the guidelines set by the National Institutes of Health (NIH publication \#85-23, revised 1985) and were approved by our Institutional Animal Care and Use Committee. The surgical procedures were similar to those published earlier [27, 28]. Briefly, after an overnight fast, animals were anesthetized using an intramuscular injection of ketamine: xylazine $(80: 10 \mathrm{mg} / \mathrm{kg})$. Ischemia $(n=6)$ was induced by occluding the portal triad (portal vein, hepatic artery, and bile duct) connected to the left and median lobes of the liver for $60 \mathrm{~min}$, leaving the blood supply to the right and caudate lobes uninterrupted. Sham-operated rats $(n=6)$ underwent the same procedure, except for clamping the portal triad. During the ischemic period, the body temperature of the rats was maintained at $37^{\circ} \mathrm{C}$ using a heating platform and lamp. At the end of the ischemic period, liver was perfused with cold saline and immediately homogenized in methanol containing 0.01 M BHT and internal standards. The resultant supernatant was separated and stored at $-80^{\circ} \mathrm{C}$ until analysis within one week.

\subsection{Statistical analysis}

The data for the stability of final samples in the autosampler and stability of the methanolic extracts in the freezer were analyzed by repeated-measure, one-way ANOVA. Ordinary, one-way ANOVA was used for the analysis of the effects of sample handling on the final results. In both cases, post-hoc analysis of means was carried out using Dunnett's multiple 
comparison test. Concentrations of the analytes in the liver samples of Sham and Ischemia groups were compared using an unpaired, two-tailed t-test with Welch's correction for the differences in the variances. In all cases, a $P$ value of $<0.05$ was considered significant. The data are presented as mean \pm S.D.

\section{Results}

\subsection{UHPLC-MS/MS}

Figures 2 and 3 represent the chromatograms of standard solutions of AA metabolites and AA, respectively, along with their internal standards. Except for 8,9-DHET, 14,15-EET, and the 14,15-EET- $\mathrm{d}_{11}$ internal standard, all the analytes showed one major peak using the MRM mode at the selected $\mathrm{m} / \mathrm{z}$ transition (Table 1) optimized for them (Fig. 2). However, at the optimized $m / z$ transition for 8,9 -DHET $(337 \rightarrow 127)$, an additional peak was observed with a retention time corresponding to 14,15 -DHET, and for 14,15-EET $(\mathrm{m} / \mathrm{z}$ transition of $319 \rightarrow 219)$, an additional peak was observed with a retention time corresponding to 8,9-EET (Fig. 2). Additionally, for $14,15-E E T-d_{11}$, with an optimized $m / z$ transition of $330 \rightarrow 268$, an additional peak was observed with a retention time corresponding to the $8,9-E E T-d_{11}$ internal standard (Fig. 2). These additional peaks were due to the fact that the optimized $\mathrm{m} / \mathrm{z}$ transitions selected for 8,9 -DHET, 14-15-EET, and 14,15-EET-d $\mathrm{d}_{11}$ were also produced by 14,15-DHET, 8,9-EET, and 8,9-EET-d 11 , respectively, although with much lower abundance. Indeed, the extra peaks were absent when 8,9-DHET, 14,15-EET, or 14,15-EET- $\mathrm{d}_{11}$ was injected alone or in the presence of all the other analytes except for 14,15-DHET, 8,9-EET or 8,9-EET-d ${ }_{11}$ (data not shown). Nevertheless, because of complete resolution of the extra peaks from the analytes of interest (Fig. 2), the extra peaks did not interfere with the quantitation of these analytes. This is in agreement with a 
previous report [29] indicating that, because of similar $\mathrm{m} / \mathrm{z}$ transitions, some fatty acids and eicosanoids need to be chromatographically resolved.

Using the gradient system with an upper limit of $60 \%$ acetonitrile, described in the Methods section, all the P450 metabolites of AA eluted within 3 min after the injection, with DHETs eluting first, followed by HETEs and EETs (Fig. 2). Whereas the retention times of DHETs ranged from 0.87 to $1.17 \mathrm{~min}$, those of HETEs and EETs ranged from 1.17 to 1.21 and from 2.21 to $2.54 \mathrm{~min}$, respectively (Fig. 2). Additionally, the retention times of the internal standards were close to those of their non-deuterated counterparts (Fig. 2). Using the isocratic mobile phase, AA and its deuterated internal standard were eluted at 0.77 and $0.76 \mathrm{~min}$, respectively (Fig. 3). The inter-day ( $n=5)$ R.S.D values for retention times were $\leq 0.8 \%$ for AA and its IS and $\leq 0.6 \%$ for the metabolites and their internal standards.

\subsection{Method validation}

The standard curves, which were prepared in ethanol, were evaluated by linear regression analysis, and the representative equations for the analytes are presented in Table 2. The coefficient of determination $\left(r^{2}\right)$ for each of the analytes was $\geq 0.98$, indicating satisfactory linearity of the standard curves in the selected calibration range. Additionally, the intercept value in the case of all analytes contributed $<15 \%$ to the lowest concentration in the calibration curve.

The results of intra- and inter-run accuracy and precision evaluated by analyzing the QC samples at low, middle, and high concentrations are presented in Table 3. The concentrations chosen for the evaluation of accuracy and precision for different analytes were based on their endogenous concentration range. The accuracy and precision values for all the 11 analytes were within the acceptable range [30]. This means that the accuracy values were within $85 \%$ to $115 \%$ for the middle and high concentrations and within $80 \%$ to $120 \%$ for the lowest concentrations 
(Table 3). Additionally, the precision values (R.S.D.) were $\leq 15 \%$ and $\leq 20 \%$ for the middle and high concentrations and the lowest concentrations, respectively (Table 3). Based on these data, the lowest concentrations listed in Table 3 were considered as the LLOQ of the assay for each analyte. Based on a 3- $\mu \mathrm{L}$ injection, these concentrations translate to a range of 0.57 (for 5,6DHET) to 5.64 (for 14,15-DHET) pg on column for various metabolites of AA.

\subsection{Recovery and matrix effect}

The recoveries of AA, P450 metabolites, and internal standards along with the matrix factor are reported in Table 4. Overall, the recovery values varied from $78.8 \%$ to $92.1 \%$ for all the compounds (Table 4). Whereas the recoveries were close to $90 \%$ for AA, HETEs, DHETs, and their corresponding internal standards, those of EETs and their internal standards were closer to $80 \%$ (Table 4). However, within each group of analytes, the recovery of all the regioselective isomers and internal standards were very close to each other (Table 4).

As for the matrix effect, the data in Table 4 indicate that the effects of matrix on the detector response to most of the analytes were minimal. The highest matrix effect was observed for 8,9-EET (MF of 82.5\%) and 11,12-EET (MF of 77.1\%), which was similar to the matrix effect for their internal standard 8-9-EET- $\mathrm{d}_{11}(\mathrm{MF}$ of $77.0 \%)$ (Table 4). Additionally, the internal standards for AA, HETEs, and DHETs showed similar extent of ion suppression as their respective standards (Table 4).

\subsection{Effects of sample handling on the liver concentrations of AA and its metabolites}

Figure 4 depicts the concentrations of AA and its metabolites in fresh liver samples that were homogenized after being kept on ice for up to 60 min and also when the samples were homogenized after a freeze-thaw cycle. The sample handling method had a significant $(P<$ 
0.001) effect on the measured concentrations of the analytes. Although the short storage of the fresh samples on ice over 60 min generally resulted in relatively modest increases in the liver concentrations of all analytes, the increases after the freeze-thaw cycle were immense. The magnitude of the increases in the analyte concentrations after the freeze-thaw cycle ranged from 10- (for 14,15-DHET) to 120- (for 5,6-EET) fold (Fig. 4).

\subsection{Stability of the methanolic extracts at $-80^{\circ} \mathrm{C}$}

Figure 5 depicts the effects of storage of methanolic extracts at $-80^{\circ} \mathrm{C}$ on the concentrations of AA and its metabolites in liver samples from six different sham-operated rats. Except for 20-HETE and 14,15-EET, the concentrations of the analytes remained constant, compared with the baseline values, during the entire four weeks of storage (Fig. 5). For 20HETE and 14,15-EET, the concentrations at the fourth week of storage were $13.5 \%(P<0.01)$ and 18.2\% $(P<0.01)$ lower than their baseline concentrations, respectively, but remained unchanged for up to two weeks of storage (Fig. 5).

\subsection{Stability of the processed samples in autosampler}

Figure 6 demonstrates the absolute peak areas of the analytes and internal standards in samples, prepared from four individual rat livers, after $0,1,3$, and $5 \mathrm{~h}$ storage $\left(4^{\circ} \mathrm{C}\right)$ in the autosampler. Neither peak areas (Fig. 6) nor analyte: IS peak area ratios (data not shown) were significantly affected by the autosampler storage for at least $5 \mathrm{~h}$ when the autosampler was maintained at $4^{\circ} \mathrm{C}$.

\subsection{Application of the method}

Figure 7 depicts the concentrations of AA and its P450 metabolites in the liver samples collected from the animals that underwent partial hepatic ischemia or sham operation. All the 11 
analytes, for which the assay was validated, were measurable in the livers of both Sham and Ischemia rats (Fig. 7). Additionally, except for 20-HETE (Fig. 7B), the concentrations of AA and its P450 metabolites in the ischemic livers were significantly higher than those in the sham groups (Fig. 7). Among the studied analytes, the highest liver concentration was observed for AA, ranging from 75 to $223 \mu \mathrm{g} / \mathrm{g}$ (Fig. 7A). Comparatively, 5,6-, 8,9-, and 11,12-EET (Fig. 7C) and 5,6-DHET (Fig. 7D) were present in very low concentrations ranging from 0.61-7.7 ng/g, with 5,6-DHET (Fig. 7D) exhibiting the lowest concentration. In comparison to 19-HETE, 20HETE showed 1.2-4 fold higher concentration (Fig. 7B). Additionally, among EETs, 14,15-EET showed the highest concentration (Fig. 7C). Similarly, among DHETs, the concentrations of 14,15-DHET were the highest (Fig. 7D). Indeed, among all the metabolites of AA, 14,15-DHET showed the highest concentrations in both the Sham and Ischemia groups (Fig. 7D).

\section{Discussion}

Earlier analytical methods for determination of cytochrome P450 metabolites of AA were based on gas chromatography coupled with mass spectrometry $[16,17,31]$. However, these methods require sample derivatization before analysis and have relatively long run times. For example, before GC-MS analysis of 20-HETE, EETs, and DHETs in dog plasma, the carboxylic and/or hydroxyl groups of these analytes had to be derivatized in two separate steps [16]. In recent years, LC-MS/MS method with electrospray ionization has emerged as a method of choice for quantitation of these metabolites and other eicosanoids [22-26, 32-34] because of lack of need for derivatization, ease of ionization, specificity, and sensitivity. However, only a limited number of the available LC-MS/MS methods have been applied and validated for the analysis of low concentrations of these metabolites in plasma [24] or tissues [22, 23]. The two available 
methods for tissues measure some EETs, HETEs, or DHETs in the human intrauterine tissues [22] or human CSF and rat brain [23]. However, there is no such quantitative method available for the determination of these metabolites in the liver, which is a major site for the P450mediated metabolism of AA [35]. Therefore, we developed and validated a sensitive method for the simultaneous quantitation of free (i.e., not bound to phospholipids) concentrations of major P450 metabolites of AA in the liver, which is reported here.

While it is also important to quantitate the free concentrations of AA along with its metabolites in order to study the effects of various pathophysiological conditions on the disposition and effects of AA metabolites, most reported LC-MS/MS methods only quantitate the AA metabolites without quantitating AA itself. This is most likely due to the significantly higher lipophilicity and basal tissue concentrations of AA, compared with its P450 metabolites. Very recently [24], an LC-MS/MS method was reported for the simultaneous analysis of AA and 32 related metabolites in human plasma using a conventional LC system with a run time of 30 min. However, the actual plasma concentrations of AA in the human plasma samples (1143 $\mathrm{ng} / \mathrm{mL})$ were substantially higher than the highest calibration standard $(200 \mathrm{ng} / \mathrm{mL})$ and the highest validated QC sample $(32 \mathrm{ng} / \mathrm{mL})$ for AA. Additionally, the recovery of AA from the samples was not reported [24]. The much higher concentrations of AA in the actual samples relative to the calibration curve implies that the samples needed to be diluted before analysis for quantitation of AA, requiring duplicate sample preparation or analysis. Therefore, it appears that there is no validated LC-MS/MS method that can quantitate very high concentrations of AA along with very low concentrations of most of its P450 metabolites in one run.

During method development process, we first tried to quantitate AA and its metabolites simultaneously in one run by developing 1) a single sample processing method to simultaneously 
recover both AA and its metabolites quantitatively from the liver, 2) a gradient mobile phase to elute AA and its metabolites in a reasonably short time, and 3) an MS setting to accurately quantitate all the analytes. Although we were successful in achieving the first (Table 4) and second (data not shown) goals, the more than 1000 fold difference in the basal concentrations of AA and its P450 metabolites in the liver (Fig. 7) prevented accurate measurement and validation of the analysis of all analytes in one run. Therefore, we injected each processed liver sample twice, once undiluted for the measurement of low concentrations of AA metabolites and the second time for the quantitation of high concentrations of AA after 4000 fold dilution of the same processed sample. Although requiring separate injections, this method is time saving as it only needs one sample preparation. Nevertheless, the gradient elution of AA metabolites using our UHPLC system resulted in satisfactory resolution of all the 10 studied metabolites within 2.5 min with a total run time of 5 min (Fig. 2). Additionally, the isocratic elution of AA occurred in $<1$ min (Fig. 3) with a run time of 2 min. Therefore, the total run time for the analysis of both AA and its metabolites separately is $\sim 7 \mathrm{~min}$, which is significantly shorter than the run time of most of the available LC-MS/MS methods that use conventional LC systems with relatively long columns [22, 24, 25, 32-34].

Measurement of the free concentrations of P450-mediated metabolites of AA in the liver is challenging. First, most of these metabolites are unstable and subject to oxidation during the sample preparation. Therefore, most methods that attempt at quantitating these metabolites add an antioxidant such as triphenylphosphine [9] or BHT $[22,23]$ to the samples to prevent oxidation during the in vitro processing of the samples before injection. Despite the addition of antioxidants, long sample preparation procedures may result in some inaccuracies in the determination of the actual concentrations of these metabolites, which is particularly true for the 
more labile metabolites, such as 5,6-EET [36]. Almost all the available methods for quantitation of P450 metabolites of AA in biological samples use solid-phase extraction [22-24], which requires multistep wash and elution of the samples followed by evaporation of the eluted samples before injection. These methods at times result in low recovery of some of the metabolites of AA [34]. Instead, our method uses a short sample preparation method consisting of direct homogenization of the samples in methanol, followed by evaporation and injection of the reconstituted residues. Indeed, our simple sample preparation method resulted in high recoveries in the range of $78.8 \%$ to $90.7 \%$ for EETs, DHETs, and HETEs (Table 4). Additionally, the recovery of the more labile 5,6-EET $(80.3 \%)$ was not different from that of other EETs (Table 4). These data suggest suitability of our sample preparation method for optimal recovery and stability of the analytes.

Another challenge in quantitation of P450 metabolites of AA in tissues is the fact that similar to AA, most of the metabolites of AA in the tissues are also bound to phospholipids, and only a small fraction of the metabolites in the tissue are in the form of free acids. Karara et al. [9] showed that $>90 \%$ of the rat liver EETs are present in phospholipids, mostly in the $s n-2$ position, and $\sim 8 \%$ are incorporated into the diglycerides and neutral lipids. They showed that free EETs constitute only a negligible fraction $(<1 \%)$ of the total EET pool of the liver tissue. The phospholipid incorporation also occurs for other metabolites of AA, such as HETEs [37, 38]. The significant incorporation of AA and its P450 metabolites in the phospholipids necessitates a sample handling method that prevents or minimizes the release of phospholipid-bound compounds during the sample storage and preparation. Otherwise, the measured quantities may not be a reflection of true concentrations of the free analytes in the tissue. 
With the exception of a report by Karara at al. [17], who used fresh liver samples, most sample handling methods reported in the literature use frozen samples for the analysis of AA and its metabolites [21,22]. We also initially used pulverized liver samples, which were prepared after snap freezing the liver tissue immediately after collection. The pulverized samples were then used for homogenization. However, this procedure resulted in substantial variability in the measured concentrations of the analytes from the same liver sample. Therefore, we tested the effects of sample handling on the liver concentrations of AA and its metabolites, which showed that freeze-thaw of the samples substantially (up to 120 fold) increases the concentrations of AA and all of its studied metabolites, relative to the fresh liver samples (Fig. 4). The freeze-thawinduced substantial increases in the concentrations of analytes suggest destabilization of phospholipids and release of AA and its metabolites from these storage sites. These results clearly show that for accurate estimation of free AA and its metabolites in tissue samples, the tissues must be processed immediately after collection without freezing.

Although the P450-mediated metabolites of AA may potentially play a significant role in the pathophysiology of some liver diseases $[15,39,40]$, we are aware of only one study that reported the free concentrations of EETs in the liver [9]. Using fresh livers and a GC/MS method, these authors reported a liver concentration of $2 \mathrm{ng} / \mathrm{g}$ for EETs as free acids. However, the concentrations of individual regioisomers of EET or other P450 mediated metabolites of AA were not reported. Others [41] have reported the liver concentrations of 8,9-, 11,12-, and 14,15DHET and 20-HETE in rats after LC/MS analysis of frozen liver samples, but were unable to detect the liver concentrations of EETs. Therefore, our study appears to be the first report of the free concentrations of the individual regioisomers of EET and DHET, in addition to 19- and 20HETE, in the liver tissue. 
In contrast to the paucity of data for the free concentrations of AA metabolites in the liver tissue, few reports exist that describe the total (free plus membrane bound) concentrations of some of the P450-mediated metabolites of AA in the liver after saponification. Using GC/MS, Karara et al. [17] reported the total concentrations of 8,9-, 11,12-, and 14,15-EET in the liver of rats. However, because of its lability, the concentration of 5,6-EET was not reported. These authors showed that the rat liver contains $\sim 0.8 \mu \mathrm{g}$ of EET/g of tissue, which is in agreement with another report from the same group [9]. In another study using a combination of HPLC and GC/MS, Zeldin et al. [42] reported that the total concentrations of 8,9-, 11,12-, and 14,15-EET in the human liver tissue were 73,113 , and $197 \mathrm{ng} / \mathrm{g}$, respectively. They were also able to quantitate all the four regioisomers of DHETs in the liver [42]. Although informative, the total concentrations of EETs may not reflect subtle temporal changes in different pathophysiologic conditions, which may affect the free concentrations of these metabolites.

The application of the assay to the measurement of AA and its metabolites (Fig. 7) indicated that partial liver ischemia significantly increases the free concentrations of AA and all of the measured metabolites except for 20-HETE. The ischemia-induced significant increase in the concentrations of AA (Fig. 7A) is most likely due to its release from the membrane storage sites during ischemia. The ischemia-induced increase in the concentrations of the metabolites of AA could potentially be due to higher formation of the metabolites, because of the availability of more substrate (AA), and/or a simultaneous release of the preformed metabolites from their storage sites. Because our model consisted of $1 \mathrm{~h}$ ischemia without any reperfusion, a significant contribution of metabolism of the released AA to the metabolites is unlikely. Therefore, the observed higher concentrations of the metabolites in ischemic livers is most likely due to their release from their storage sites. Indeed, as mentioned before, both EETs [9] and HETEs [37, 38] 
are incorporated into the rat liver phospholipids and are released from these sites by the action of phospholipases $[9,10]$. Furthermore, the activity and/or concentration of phospholipases are expected to increase as a result of ischemic injury [43, 44], potentially releasing the metabolites from their storage sites. Nevertheless, further studies are needed to determine the exact mechanisms responsible for the ischemia-induced increases in the liver concentration of the AA metabolites observed in our study (Fig. 7).

\section{Conclusions}

In conclusion, a rapid and sensitive UHPLC-MS/MS method was developed for the quantitation of free concentrations of AA and its major P450 metabolites in the liver. After a single sample preparation procedure, the processed sample is injected twice, once for the simultaneous quantitation of all four regioisomers of EET and DHET and two major regioisomers of HETE, in addition to their respective deuterated internal standards, and the second time for the analysis of AA. The method was successfully applied to quantitation of the liver concentrations of AA and its metabolites in rats subjected to partial hepatic ischemia.

\section{Acknowledgements}

This work was supported by a grant from Texas Higher Education Coordinating Board (NHARP Program). The authors would like to sincerely thank Drs. Li Li and Helen Thorsheim for training V.E. to use the LC-MS/MS system and for technical assistance during the development of this assay. 


\section{References}

[1] R. Kaspera, R.A. Totah, Expert Opin. Drug Metab. Toxicol. 5 (2009) 757.

[2] A.A. Caro, A.I. Cederbaum, Free Radic. Biol. Med. 40 (2006) 364.

[3] W.B. Campbell, J.R. Falck, Hypertension 49 (2007) 590.

[4] J.H. Capdevila, J.R. Falck, R.C. Harris, J. Lipid Res. 41 (2000) 163.

[5] R.J. Roman, Physiol. Rev. 82 (2002) 131.

[6] J. Capdevila, L.J. Marnett, N. Chacos, R.A. Prough, R.W. Estabrook, Proc. Natl. Acad. Sci. U S A $79(1982) 767$.

[7] A.R. Morrison, N. Pascoe, Proc. Natl. Acad. Sci. U S A 78 (1981) 7375.

[8] E.H. Oliw, J.A. Lawson, A.R. Brash, J.A. Oates, J. Biol. Chem. 256 (1981) 9924.

[9] A. Karara, E. Dishman, J.R. Falck, J.H. Capdevila, J. Biol. Chem. 266 (1991) 7561.

[10] A.A. Spector, X. Fang, G.D. Snyder, N.L. Weintraub, Prog. Lipid Res. 43 (2004) 55.

[11] V. Randriamboavonjy, R. Busse, I. Fleming, Hypertension 41 (2003) 801.

[12] B. Fisslthaler, R. Popp, L. Kiss, M. Potente, D.R. Harder, I. Fleming, R. Busse, Nature 401 (1999) 493.

[13] A.O. Oyekan, J. Pharmacol. Exp. Ther. 313 (2005) 1289.

[14] G.J. Gross, A. Hsu, J.R. Falck, K. Nithipatikom, J. Mol. Cell. Cardiol. 42 (2007) 687.

[15] D. Sacerdoti, H. Jiang, S. Gaiani, J.C. McGiff, A. Gatta, M. Bolognesi, Prostaglandins Other Lipid Mediat. 96 (2011) 72.

[16] K. Nithipatikom, R.F. DiCamelli, S. Kohler, R.J. Gumina, J.R. Falck, W.B. Campbell, G.J. Gross, Anal. Biochem. 292 (2001) 115.

[17] A. Karara, E. Dishman, I. Blair, J.R. Falck, J.H. Capdevila, J. Biol. Chem. 264 (1989) 19822. 
[18] K.G. Maier, L. Henderson, J. Narayanan, M. Alonso-Galicia, J.R. Falck, R.J. Roman, Am. J. Physiol. Heart Circ. Physiol. 279 (2000) H863.

[19] H. Yue, K.I. Strauss, M.R. Borenstein, M.F. Barbe, L.J. Rossi, S.A. Jansen, J. Chromatogr. B 803 (2004) 267.

[20] K. Nithipatikom, A.J. Grall, B.B. Holmes, D.R. Harder, J.R. Falck, W.B. Campbell, Anal. Biochem. 298 (2001) 327.

[21] H. Yue, S.A. Jansen, K.I. Strauss, M.R. Borenstein, M.F. Barbe, L.J. Rossi, E. Murphy, J. Pharm. Biomed. Anal. 43 (2007) 1122.

[22] J.H. Zhang, T. Pearson, B. Matharoo-Ball, C.A. Ortori, A.Y. Warren, R. Khan, D.A. Barrett, Anal. Biochem. 365 (2007) 40.

[23] T.M. Miller, M.K. Donnelly, E.A. Crago, D.M. Roman, P.R. Sherwood, M.B. Horowitz, S.M. Poloyac, J. Chromatogr. B 877 (2009) 3991.

[24] D.D. Shinde, K.B. Kim, K.S. Oh, N. Abdalla, K.H. Liu, S.K. Bae, J.H. Shon, H.S. Kim, D.H. Kim, J.G. Shin, J. Chromatogr. B 911 (2012) 113.

[25] R. Deems, M.W. Buczynski, R. Bowers-Gentry, R. Harkewicz, E.A. Dennis, Methods Enzymol. 432 (2007) 59.

[26] R.C. Murphy, R.M. Barkley, K. Zemski Berry, J. Hankin, K. Harrison, C. Johnson, J. Krank, A. McAnoy, C. Uhlson, S. Zarini, Anal. Biochem. 346 (2005) 1.

[27] I.H. Shaik, R. Mehvar, J. Pharm. Sci. 100 (2011) 5281.

[28] R. Parasrampuria, I.H. Shaik, R. Mehvar, J. Pharm. Pharm. Sci. 15 (2012) 318.

[29] L. Kortz, J. Dorow, S. Becker, J. Thiery, U. Ceglarek, J. Chromatogr. B 927 (2013) 209. 
[30] V.P. Shah, K.K. Midha, J.W. Findlay, H.M. Hill, J.D. Hulse, I.J. McGilveray, G. McKay, K.J. Miller, R.N. Patnaik, M.L. Powell, A. Tonelli, C.T. Viswanathan, A. Yacobi, Pharm. Res. 17 (2000) 1551.

[31] D. Tsikas, J. Chromatogr. B: Biomed. Sci. Appl. 717 (1998) 201.

[32] J.W. Newman, T. Watanabe, B.D. Hammock, J. Lipid Res. 43 (2002) 1563.

[33] S.L. Lundstrom, F.L. D'Alexandri, K. Nithipatikom, J.Z. Haeggstrom, A.M. Wheelock, C.E. Wheelock, Methods Mol. Biol. 579 (2009) 161.

[34] R. Martin-Venegas, R. Casillas, O. Jauregui, J.J. Moreno, J. Pharm. Biomed. Anal. 56 (2011) 976.

[35] A.A. El-Sherbeni, M.E. Aboutabl, B.N. Zordoky, A. Anwar-Mohamed, A.O. El-Kadi, AAPS J. (2012).

[36] D. Fulton, J.R. Falck, J.C. McGiff, M.A. Carroll, J. Quilley, J. Lipid Res. 39 (1998) 1713.

[37] C.F. Richards, W.B. Campbell, Prostaglandins 38 (1989) 565.

[38] M.A. Carroll, M. Balazy, D.D. Huang, S. Rybalova, J.R. Falck, J.C. McGiff, Kidney Int. 51 (1997) 1696.

[39] D. Sacerdoti, M. Balazy, P. Angeli, A. Gatta, J.C. McGiff, J. Clin. Invest. 100 (1997) 1264.

[40] D. Sacerdoti, A. Gatta, J.C. McGiff, Prostaglandins Other Lipid Mediat. 72 (2003) 51.

[41] B.N. Zordoky, A. Anwar-Mohamed, M. Aboutabl, A.O. El-Kadi, Drug Metab. Dispos. (2011).

[42] D.C. Zeldin, C.R. Moomaw, N. Jesse, K.B. Tomer, J. Beetham, B.D. Hammock, S. Wu, Arch. Biochem. Biophys. 330 (1996) 87.

[43] M. Sakon, H. Ariyoshi, K. Umeshita, M. Monden, Surg. Today 32 (2002) 1. 
[44] D.R. Monbaliu, C.N. Dubuisson, M.M. Zeegers, M.M. Crabbe, J.M. Fevery, J.M. Pirenne, J.F. van Pelt, J. Surg. Res. 151 (2009) 125.s 


\section{Table 1}

Compound parameters for arachidonic acid, its P450 metabolites, and the deuterated internal standards with MRM in negative electrospray ionization mode.

\begin{tabular}{|c|c|c|c|c|c|c|}
\hline \multirow[t]{2}{*}{ Analyte } & Q1 Mass & Q3 Mass & DP & $\mathrm{CE}$ & CXP & \multirow[t]{2}{*}{ EP } \\
\hline & \multicolumn{2}{|l|}{$\mathrm{Da}$} & \multicolumn{3}{|l|}{ V } & \\
\hline AA & 303 & 259 & -125 & -18 & -15 & -10 \\
\hline $\mathrm{AA}-\mathrm{d}_{8}$ & 311 & 267 & -125 & -18 & -15 & -10 \\
\hline 19-HETE & 319 & 231 & -125 & -20 & -13 & -10 \\
\hline 20-HETE & 319 & 245 & -95 & -22 & -15 & -10 \\
\hline 20-HETE-d 6 & 325 & 281 & -110 & -22 & -15.5 & -10 \\
\hline 5,6-EET & 319 & 191 & -30 & -14 & -11 & -10 \\
\hline 8,9-EET & 319 & 155 & -30 & -14 & -11 & -10 \\
\hline 8,9-EET- $\mathrm{d}_{11}$ & 330 & 155 & -25 & -18 & -19 & -10 \\
\hline 11,12-EET & 319 & 208 & -120 & -16 & -13 & -10 \\
\hline 14,15-EET & 319 & 219 & -105 & -16 & -13 & -10 \\
\hline $14,15-E E T-d_{11}$ & 330 & 268 & -120 & -18 & -15 & -10 \\
\hline 5,6-DHET & 337 & 145 & -115 & -22 & -11 & -10 \\
\hline 8,9-DHET & 337 & 127 & -105 & -26 & -11 & -10 \\
\hline 11,12-DHET & 337 & 167 & -115 & -24 & -19 & -10 \\
\hline 14,15-DHET & 337 & 207 & -40 & -24 & -13 & -10 \\
\hline $14,15-$ DHET-d ${ }_{11}$ & 348 & 207 & -95 & -24 & -13 & -10 \\
\hline
\end{tabular}

Abbreviations: DP, declustering potential; CE, collision energy; CXP, collision cell exit potential; EP, entrance potential 


\section{Table 2}

Representative linear equations for the standard curves of arachidonic acid and its P450 metabolites prepared in ethanol.

\begin{tabular}{|c|c|}
\hline Analyte & Linear equation \\
\hline AA & $y=0.04540 x+0.05112$ \\
\hline 19-HETE & $y=0.03078 x-0.00172$ \\
\hline 20-HETE & $y=0.02810 x+0.00345$ \\
\hline 5,6-EET & $y=0.17962 x-7.87085 e-4$ \\
\hline 8,9-EET & $y=0.11991 x-0.00340$ \\
\hline 11,12-EET & $y=0.07174 x-0.00275$ \\
\hline 14,15-EET & $y=0.18711 x+0.00151$ \\
\hline 5,6-DHET & $y=0.00575 x-7.81621 e-5$ \\
\hline 8,9-DHET & $y=0.00393 x-2.02197 e-4$ \\
\hline 11,12-DHET & $y=0.01106 x-4.33385 e-4$ \\
\hline 14,15-DHET & $y=0.00870 x-7.74597 e-5$ \\
\hline
\end{tabular}

$\mathrm{y}$ : peak area ratio; $\mathrm{x}$ : analyte concentration in $\mathrm{ng} / \mathrm{mL}$ except for $\mathrm{AA}$, which was $\mu \mathrm{g} / \mathrm{mL}$. 
Table 3

Intra- and inter-run accuracy and precision of the method using samples prepared in ethanol $(n=5)$.

\begin{tabular}{|c|c|c|c|c|}
\hline \multirow{2}{*}{ Concentration } & \multicolumn{2}{|l|}{ Intra-run } & \multicolumn{2}{|l|}{ Inter-run } \\
\hline & Accuracy & R.S.D. (\%) & Accuracy & R.S.D. (\%) \\
\hline \multicolumn{5}{|l|}{$\mathrm{AA}(\mu \mathrm{g} / \mathrm{mL})$} \\
\hline 7.81 & 97.4 & 14.3 & 90.1 & 15.6 \\
\hline 62.5 & 104 & 2.00 & 106 & 8.35 \\
\hline 500 & 93.3 & 2.34 & 90.5 & 5.03 \\
\hline \multicolumn{5}{|c|}{ 19-HETE (ng/mL) } \\
\hline 0.94 & 85.6 & 10.7 & 110 & 10.4 \\
\hline 3.75 & 100 & 2.31 & 94.1 & 8.12 \\
\hline 30 & 97.2 & 4.29 & 97.8 & 4.17 \\
\hline \multicolumn{5}{|c|}{ 20-HETE (ng/mL) } \\
\hline 0.94 & 95.1 & 6.02 & 98.8 & 13.7 \\
\hline 3.75 & 102 & 10.3 & 105 & 5.66 \\
\hline 30 & 95.2 & 3.13 & 100 & 5.18 \\
\hline \multicolumn{5}{|l|}{ 5, 6-EET (ng/mL) } \\
\hline 0.38 & 81.6 & 10.7 & 83.6 & 19.3 \\
\hline 1.5 & 100 & 2.41 & 99.3 & 6.13 \\
\hline 12 & 98.4 & 3.33 & 102 & 3.10 \\
\hline \multicolumn{5}{|l|}{ 8,9-EET (ng/mL) } \\
\hline 0.75 & 96.0 & 19.3 & 99.9 & 10.9 \\
\hline 3.0 & 95.9 & 8.56 & 98.6 & 5.25 \\
\hline 24 & 99.1 & 2.86 & 99.7 & 2.59 \\
\hline \multicolumn{5}{|c|}{ 11,12-EET (ng/mL) } \\
\hline 0.38 & 99.1 & 3.49 & 111 & 8.87 \\
\hline 1.5 & 102 & 3.06 & 100 & 5.63 \\
\hline 12 & 92.4 & 3.13 & 100 & 3.14 \\
\hline \multicolumn{5}{|c|}{ 14,15-EET (ng/mL) } \\
\hline 0.38 & 99.3 & 14.0 & 94.7 & 13.4 \\
\hline 1.5 & 94.7 & 7.47 & 96.6 & 9.64 \\
\hline 12 & 92.5 & 3.31 & 101 & 2.67 \\
\hline \multicolumn{5}{|c|}{ 5,6-DHET (ng/mL) } \\
\hline 0.19 & 98.6 & 11.6 & 97.8 & 10.6 \\
\hline 1.5 & 97.6 & 1.69 & 98.1 & 3.16 \\
\hline 12 & 97.6 & 2.61 & 100 & 2.02 \\
\hline \multicolumn{5}{|c|}{ 8,9-DHET (ng/mL) } \\
\hline 0.38 & 100 & 7.93 & 94.7 & 9.21 \\
\hline 3.0 & 98.2 & 2.48 & 93.0 & 7.87 \\
\hline 24 & 96.8 & 4.84 & 99.9 & 1.4 \\
\hline \multicolumn{5}{|c|}{ 11,12-DHET (ng/mL) } \\
\hline 0.48 & 106 & 3.51 & 98.6 & 3.24 \\
\hline 3.75 & 101 & 3.12 & 98.3 & 2.97 \\
\hline 30 & 98.5 & 3.22 & 100 & 1.31 \\
\hline \multicolumn{5}{|c|}{ 14,15-DHET (ng/mL) } \\
\hline 1.88 & 87.9 & 1.94 & 86.5 & 5.56 \\
\hline 15 & 106 & 2.72 & 102 & 3.48 \\
\hline 120 & 95.8 & 2.44 & 97.1 & 1.63 \\
\hline
\end{tabular}




\section{Table 4}

Recovery and matrix factor (mean \pm S.D.) of AA, its P450

metabolites, and internal standards using liver homogenates $(n=6)$.

\begin{tabular}{|c|c|c|}
\hline Analyte & $\%$ Recovery & Matrix factor \\
\hline AA & $87.5 \pm 4.1$ & $83.7 \pm 14.6$ \\
\hline AA- $d_{8}$ & $87.7 \pm 3.5$ & $92.3 \pm 6.0$ \\
\hline 19-HETE & $90.7 \pm 2.9$ & $90.0 \pm 2.8$ \\
\hline 20-HETE & $88.9 \pm 4.6$ & $91.9 \pm 3.6$ \\
\hline 20-HETE-d 6 & $92.1 \pm 5.0$ & $91.2 \pm 6.3$ \\
\hline 5,6-EET & $80.3 \pm 7.4$ & $88.7 \pm 1.5$ \\
\hline 8,9-EET & $78.8 \pm 6.6$ & $82.5 \pm 3.2$ \\
\hline $8,9-E E T-d_{11}$ & $80.0 \pm 6.8$ & $77.0 \pm 3.1$ \\
\hline 11,12-EET & $80.4 \pm 6.8$ & $77.1 \pm 2.1$ \\
\hline 14,15-EET & $82.5 \pm 5.6$ & $94.6 \pm 1.4$ \\
\hline $14,15-E E T-d_{11}$ & $83.5 \pm 5.1$ & $95.5 \pm 2.2$ \\
\hline 5,6-DHET & $87.0 \pm 3.7$ & $92.8 \pm 2.2$ \\
\hline 8,9-DHET & $88.9 \pm 3.5$ & $85.5 \pm 1.4$ \\
\hline 11,12-DHET & $87.8 \pm 5.8$ & $92.0 \pm 1.9$ \\
\hline 14,15-DHET & $90.4 \pm 4.4$ & $91.8 \pm 3.2$ \\
\hline $14,15-$ DHET-d ${ }_{11}$ & $90.6 \pm 4.7$ & $92.7 \pm 2.9$ \\
\hline
\end{tabular}




\section{Legend for Figures}

Fig. 1. Chemical structures of arachidonic acid and its metabolites that were analyzed in this study.

Fig. 2. Representative chromatograms of DHETs (Panel A), HETEs (Panel B), EETs (Panel C), and their internal standards (Panel D). The peaks correspond to $3 \mu \mathrm{L}$ of sample injected into the instrument. The analyte concentrations in the samples were $30 \mathrm{ng} / \mathrm{mL}$ of 19-HETE, 20-HETE, and 11,12-DHET; $24 \mathrm{ng} / \mathrm{mL}$ of 8,9-EET and 8,9-DHET; $12 \mathrm{ng} / \mathrm{mL}$ of 5,6-EET, 11,12-EET, 14,15-EET, and 5,6-DHET; $120 \mathrm{ng} / \mathrm{mL}$ of 14,15-DHET; $30 \mathrm{ng} / \mathrm{mL}$ of $20-H E T E-\mathrm{d}_{6} ; 25 \mathrm{ng} / \mathrm{mL}$ of 8,9-EET- $\mathrm{d}_{11}$ and 14,15-EET-d $\mathrm{d}_{11}$; and $60 \mathrm{ng} / \mathrm{mL}$ of 14,15-DHET- $\mathrm{d}_{11}$.

Fig. 3. Representative chromatograms of $A A$ and its internal standard $\left(A A-d_{8}\right)$ in a sample containing $500 \mu \mathrm{g} / \mathrm{mL}$ of AA and $70 \mu \mathrm{g} / \mathrm{mL}$ of AA- $\mathrm{d}_{8}$. The sample was diluted 4000 fold before injection.

Fig. 4. The effects of storage of fresh livers on ice for 0 (Fresh-0), 20 (Fresh-20), 40 (Fresh-40), or 60 (Fresh-60) min and sample freeze-thaw on the liver concentrations of AA and its metabolites. Columns and bars represent mean and S.D. values, respectively $(n=3) .{ }^{*}, P<0.05$; $* *, P<0.01 ; * * *, P<0.001$ : Significantly different from the Fresh-0 sample, based on one-way ANOVA, followed by post-hoc analysis using Dunnett's multiple comparison test.

Fig. 5. Effects of storage of methanolic extracts of fresh liver samples at $80^{\circ} \mathrm{C}$ on the concentrations of AA and its metabolites. Columns and bars represent mean and S.D. values, respectively $(n=6) .{ }^{*}, P<0.05 ; * *, P<0.01$ : Significantly different from the baseline concentration based on repeated measures one-way ANOVA, followed by post-hoc analysis using Dunnett's multiple comparison test. 
Fig. 6. Absolute peak areas of the analytes and internal standards after storage $\left(4^{\circ} \mathrm{C}\right)$ of the final processed samples in the autosampler for up to $5 \mathrm{~h}$. Columns and bars represent mean and S.D. values, respectively $(n=4)$. There were no statistical differences among the groups tested by repeated measure one-way ANOVA.

Fig. 7. The concentrations of arachidonic acid (A), 19- and 20-HETE (B), 5,6-, 8,9-, 11,12-, and 14,15-EET (C), 5,6-, 8,9-, 11,12-, and 14,15-DHET (D) in the liver samples from Sham and Ischemia rats. The columns and bars represent mean and S.D. values, respectively $(n=6){ }^{*}, P<$ $0.05 ;{ }^{* *}, P<0.01$ : Significantly different from the Sham group, based on unpaired, two-tailed $\mathrm{t}$ test with Welch's correction. 
Figure 1

5,6-DHET
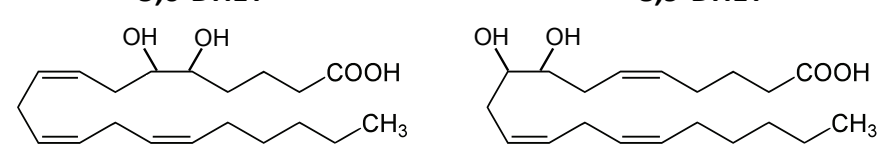

Epoxide Hydrolase
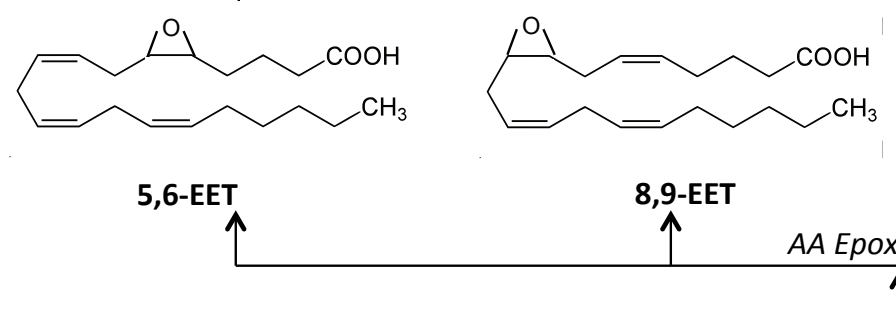

11,12-DHET

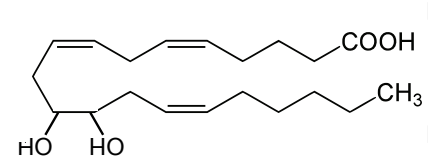

Epoxide Hydrolase

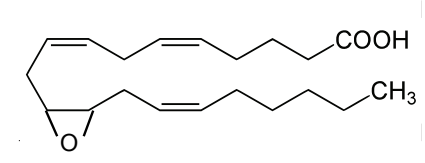

11,12, : Etr
14,15-DHET

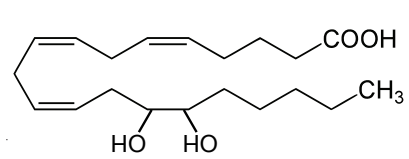

$\uparrow$ Epoxide Hydrolase

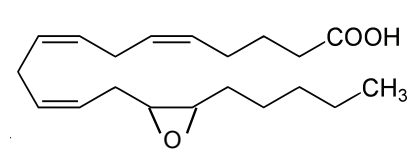

14,15-EET

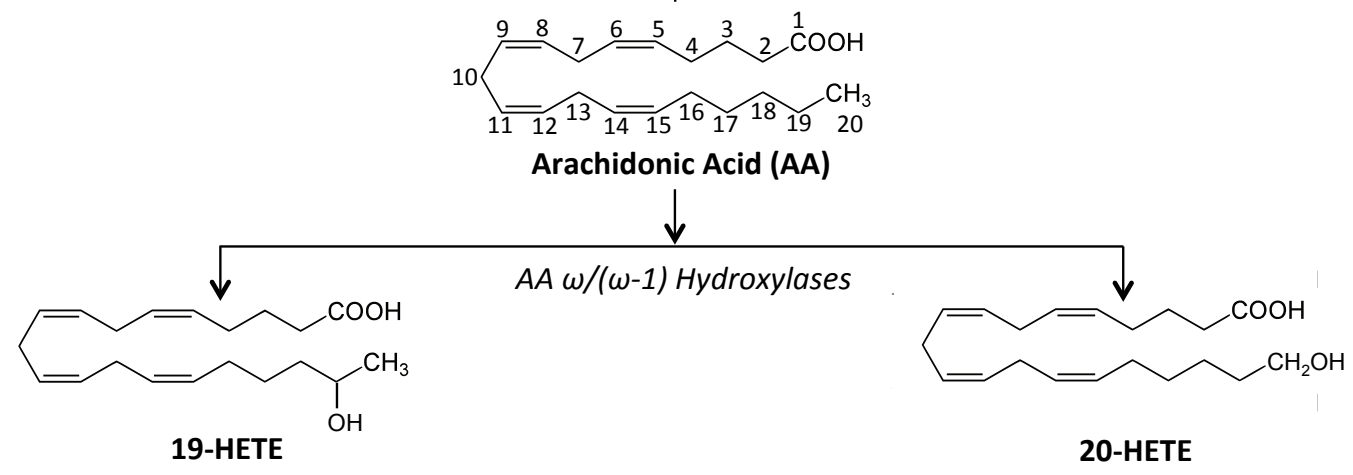




\section{A: DHETs}
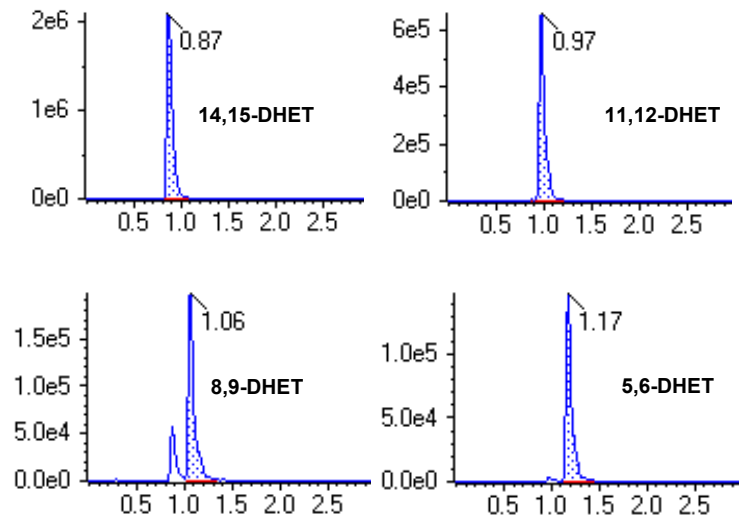

\section{B: HETEs}
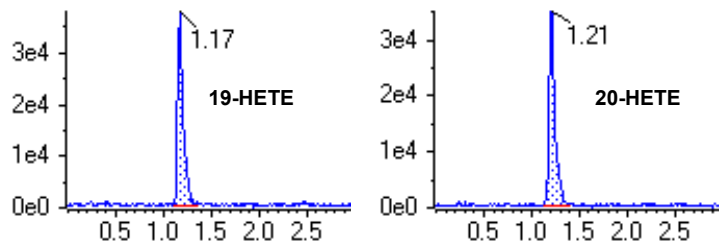

\section{C: EETs}
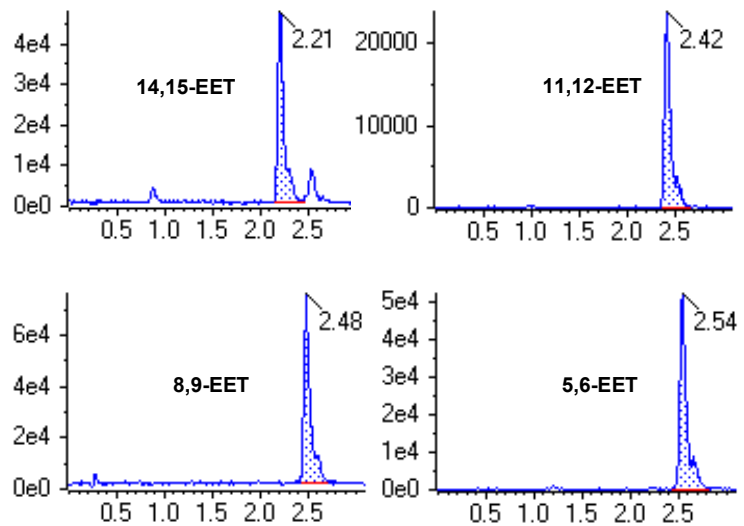

\section{D: Internal Standards}
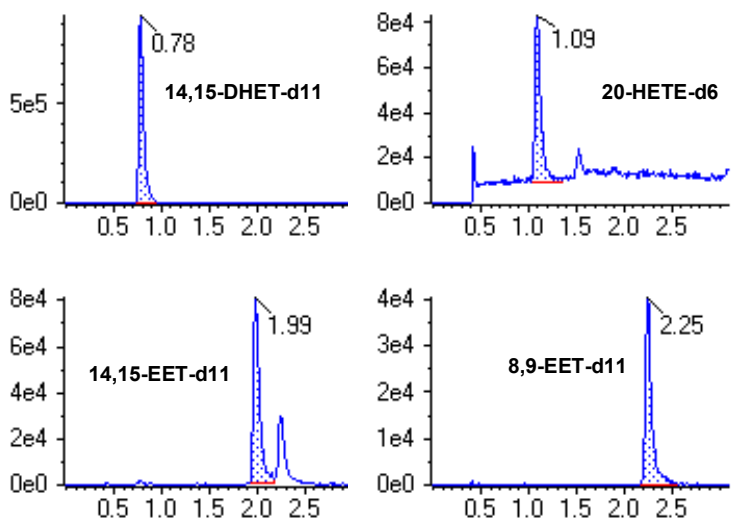

Time (Minutes) 

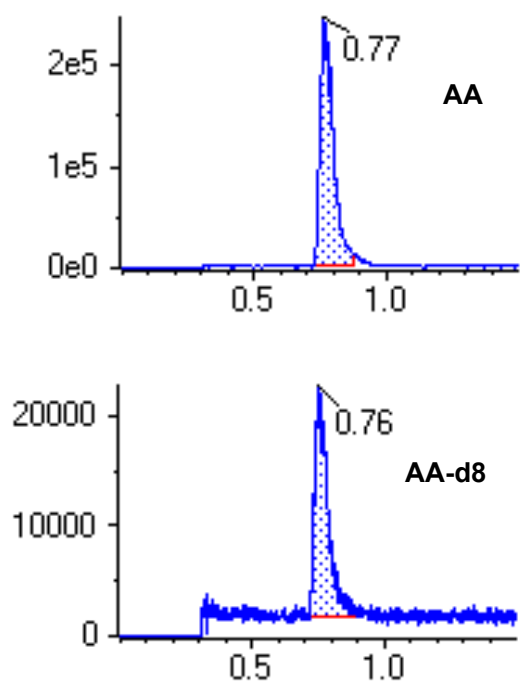

Time (Minutes)

Figure 3 


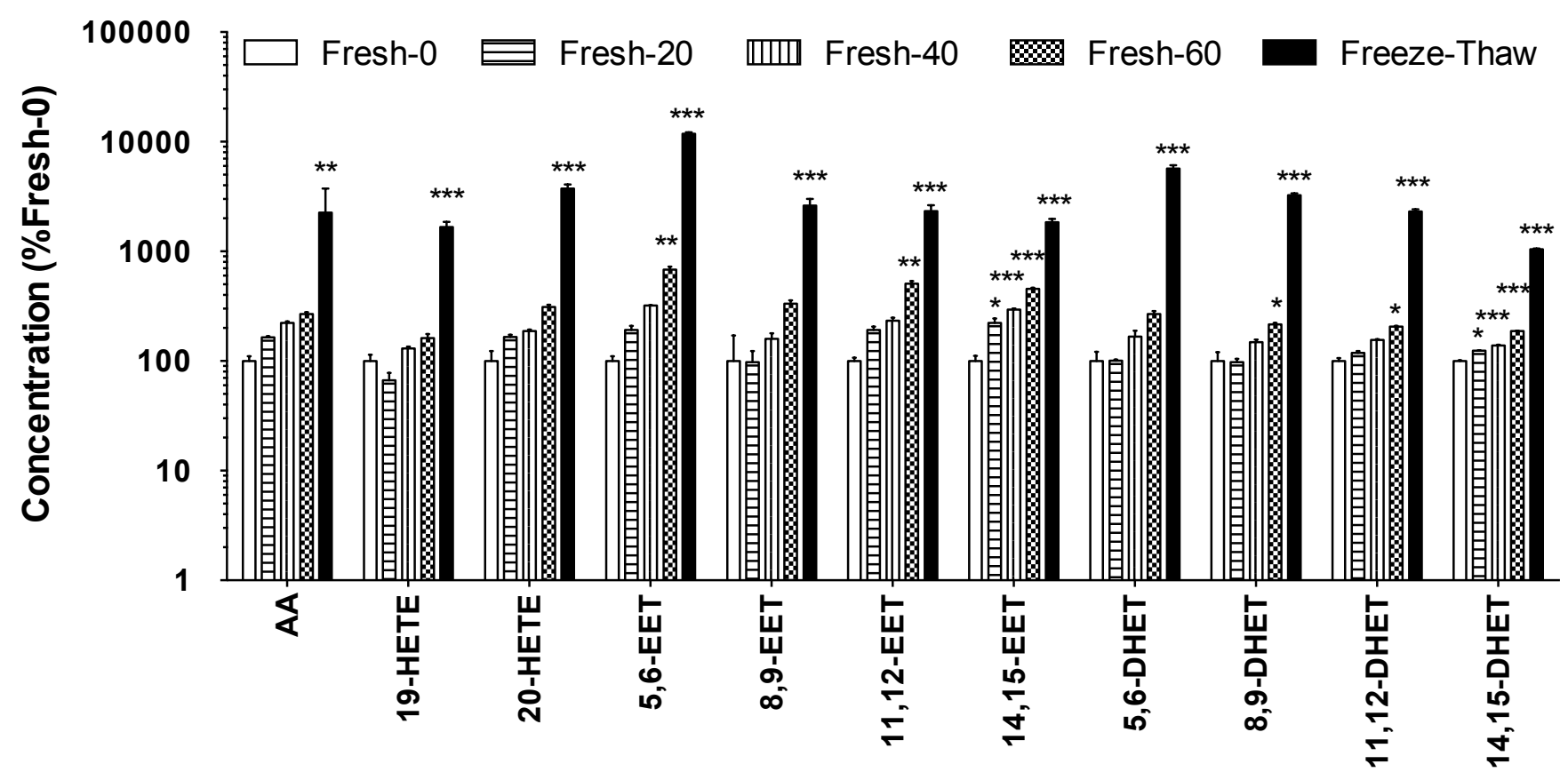


Figure 5

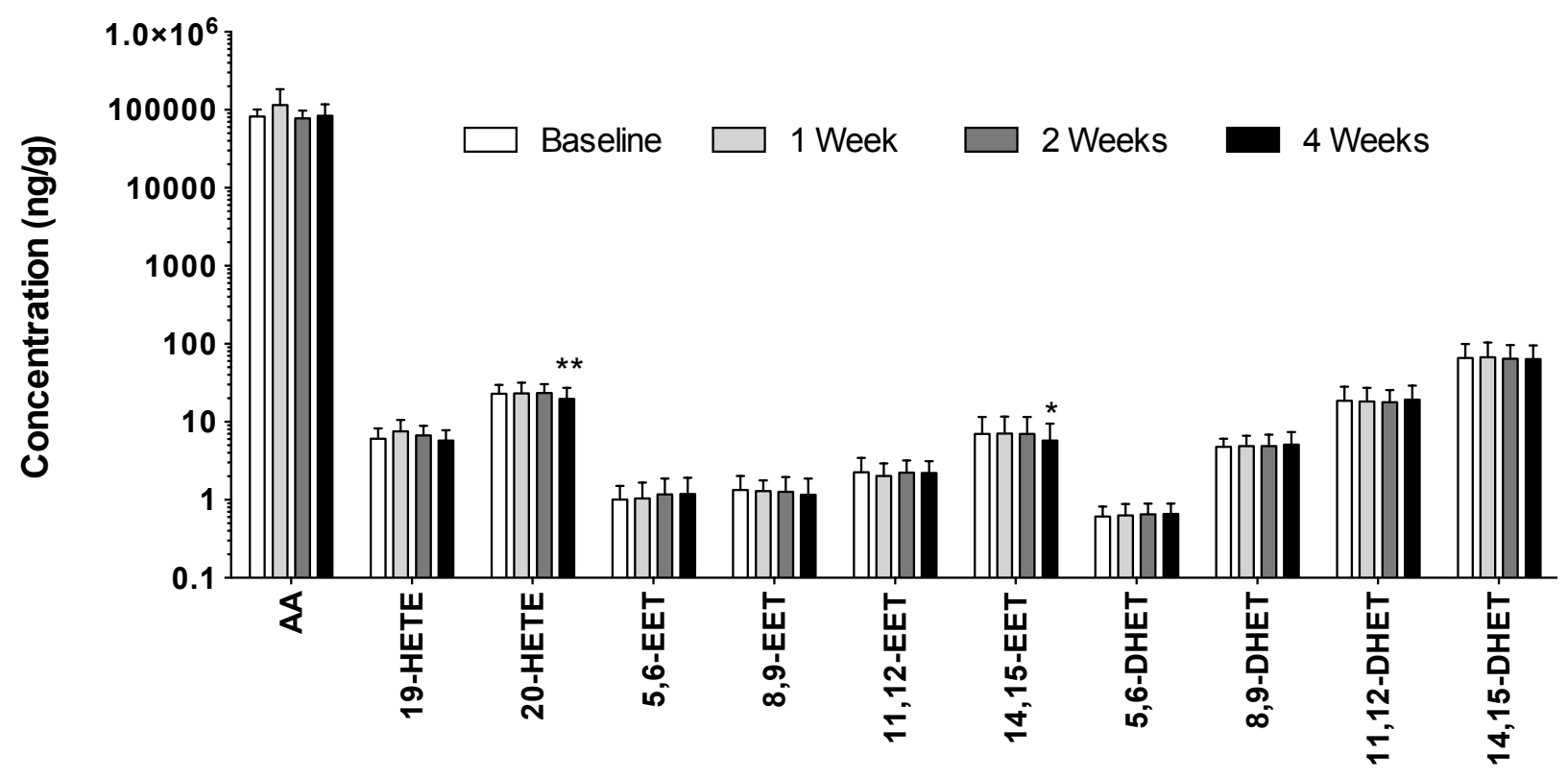


Figure 6

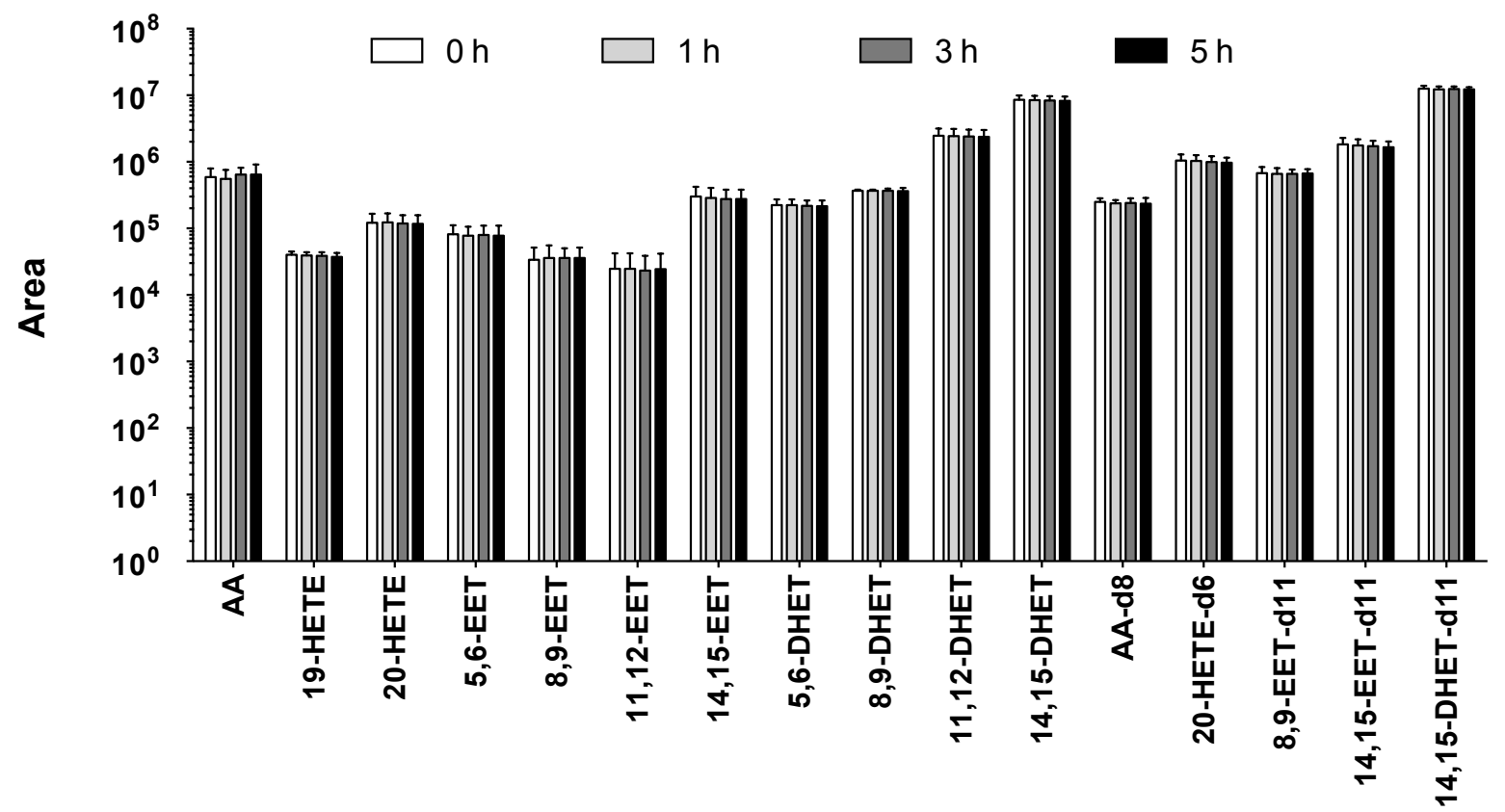




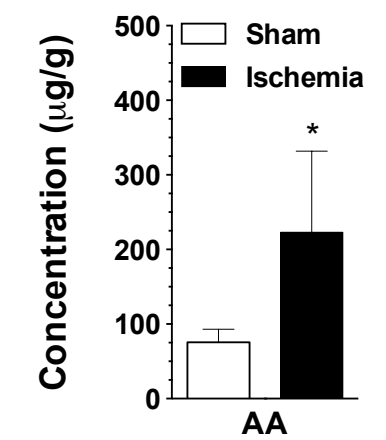

(C)

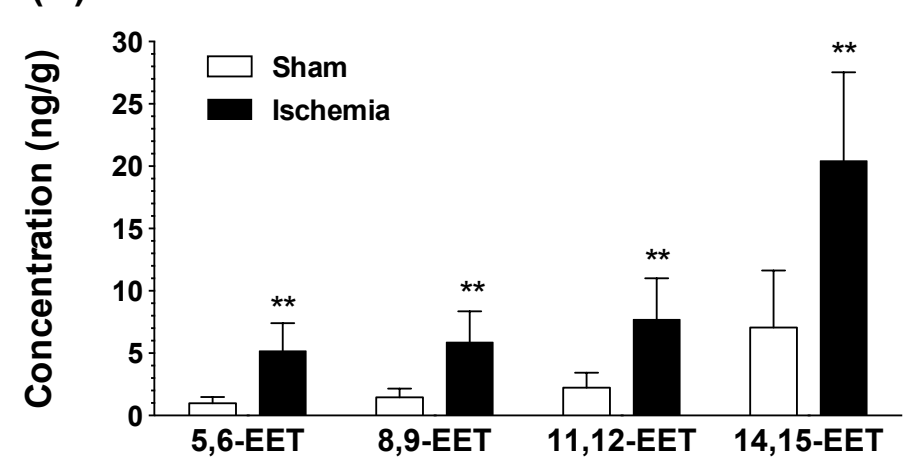

(D)

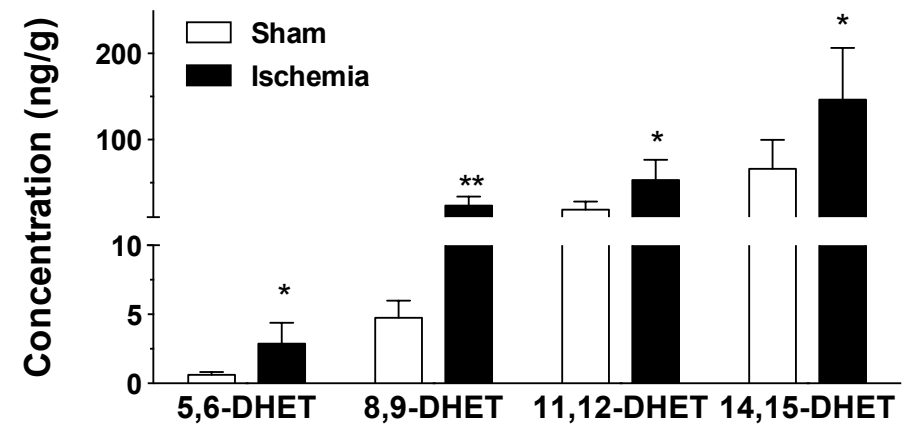

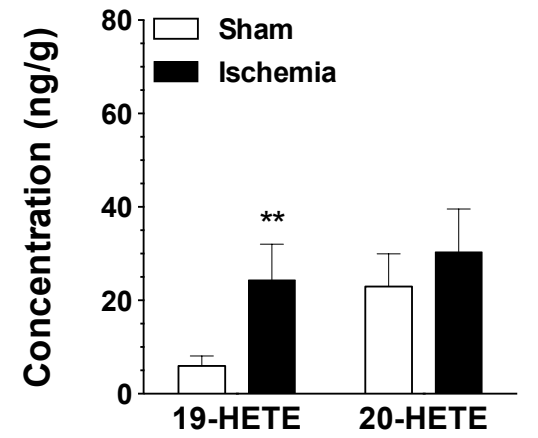

B)

$\begin{array}{ll}\text { (A) } & \text { (B) }\end{array}$

$\begin{array}{ll}\text { (A) } & \text { (B) }\end{array}$

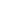

